\title{
NON-AXISYMMETRIC EFFECTS ON LONG LIQUID BRIDGES
}

\author{
J. M. Perales \\ Laboratorio de Aerodinámica, E.T.S.l. Aeronáuticos, Universidad Politécnica, 28040 Madrid, Spain
}

\begin{abstract}
The stability of long hiquid bridges under non-axisymmetric disturbances like a microgravitational force acting perpendicular to the liquid brioge axis or a non-coaxiality of the disks is analyzed through an asymptotic method based on bifurcation techniques. Results obtained indicate that such non-axisymmetric effects are of higher order than those produced by axisymmetric perturbations.
\end{abstract}

\section{INTRODUCTION}

In the last 10 years, a significant number of the. oretical and experimental papers dealing with the behavior of liquid bridges in a low gravity environment have been published. Such studies are interesting because a liquid bridge is, under a strictly mechanical point of view, similar to the fluid configuration appearing in the crystal growth process known as the floating zone technique. In most of these papers (a short review of the literature in this field can be found in Meseguer and Sanz[1]) only axisymmetric liquid bridges under axisymmetric perturbations are considered, and only in a few of them non-axisymmetric effects like the $\mathrm{C}$-mode[2,3] or gravitational forces acting perpendicular to the liquid bridge axis $[4,5]$ have been treated.

In each one of the two European missions of Spacelab (Spacelab-1 and Spacelab-D1) an experiment dealing with the stability of long liquid bridges under mechanical disturbances has been performed. One of the problems arising in the anaiysis of such experimental results is that experimental configurations were not exactly those foreseen because of a disalignment of the axes of the disks supporting the liquid column (Spacelab-1)[6]. The question to be answered is how this disalignment modifies the stability of the liquid bridge.

Theoretical analysis and experiments performed on Earth seem to indicate that non-axisymmetric perturbations are less important than axisymmetric ones. For example, Coriell et al.[4] stated that the effect of a transversal microgravity on the stability limit of a slender liquid bridge is of the order of the square of the perturbation, whereas the effects produced by axisymmetric perturbations like small volume changes, axial microgravity or a small difference in disks diameter are of the order of the perturbation in the first case and of the order of the two-thirds power of the perturbation in the second two[7].
In this paper, the stability of long liquid bridges under non-axisymmerric perturbations is analyzed by using an asymptotic method based on the idea of the bifurcation equation, already used in the case of isorotating liquid bridges by Vega and Perales[3]. This method simplifies the calculation of the equjlibrium shapes (either stable or unstable) and stability limits, allowing the knowiedge of the character of the branching points (sub- or supercritical).

The main result here obtained is that nonaxisymmetric perturbations have a negligible effect on stability limit when compared with axisymmetric ones. An additional consequence of the analysis here presented is that, in most cases, the study of the symmetries appearing in the problem is sufficient to establish the order of the modification of the maximum stable length of a liquid bridge under a given perturbation, without solving the whole problem.

\section{PROBLEM FORMULATION}

Let us consider a slender liquid bridge as sketched in Fig. 1: a liquid column held by surface tension forces between two circular disks of radius $\mathbf{R}_{0}$ placed a distance $\mathbf{L}$ apart. Both disks are parallel but non-coaxial, $2 \mathrm{E}$ being the distance between both axes. The liquid bridge is subject to a transversal gravity $\boldsymbol{g}$ and its volume is equal to that of a cylindrical column held between two coaxial disks of radius $\mathbf{R}_{0}$ at a distance $\mathbf{L}\left(\mathbf{V}=\pi \mathbf{R}_{0}^{\mathbf{L}} \mathbf{L}\right)$.

Let $\mathbf{R}=\mathbf{R}(\mathbf{Z}, \theta)$ the equation of the liquid bridge interface. If the fluid is at rest the equation governing the interface shape is obtained by expressing the equilibrium between the different pressure forces at the interface

$\sigma\left(1 / \mathbf{R}_{1}+1 / \mathbf{R}_{2}\right)+\mathbf{P}_{0}-\mathbf{P}_{a}+\rho g \mathbf{R} \cos (\theta+\alpha)=0$,

where $\alpha$ is the angle between the plane defined by the axes of the disks and the direction of transversal microgravity, $g$ the acceleration due to this gravity, $\sigma$ the surface tension, $\mathbf{R}_{1}$ and $\mathbf{R}_{2}$ the principal radii of curvature, $\rho$ the liquid density, $P_{a}$ the ambient pres- 


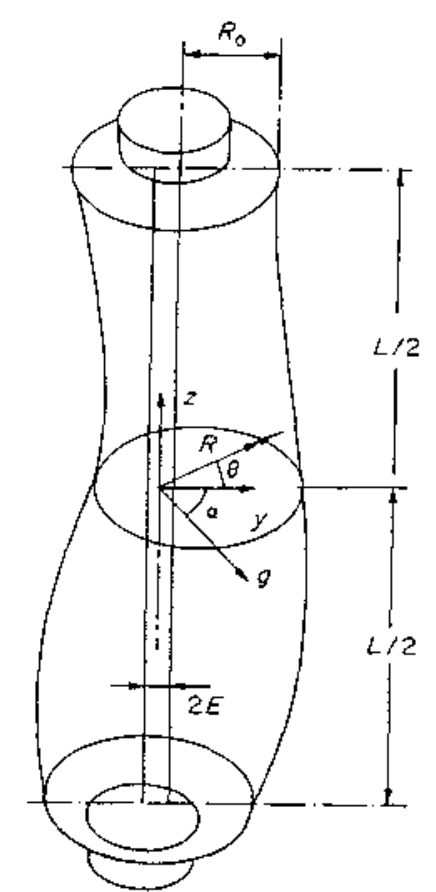

Fig. 1. Geometry and coordinate system for the perturbed liquid bridge.

sure and $P_{0}$ a yet unknown constant giving the origin of pressures inside the liquid bridge. To calculate $\mathbf{P}_{0}$ the condition that the volume of the liquid bridge remains equal to that of a cytinder must be imposed, that is

$$
\int_{-\mathbf{L} / 2}^{\mathrm{L} / 2} \mathrm{dZ} \int_{0}^{2 \pi} \mathbf{R}^{2}(Z, \theta) \mathrm{d} \theta=2 \pi \mathbf{L R}_{\theta}^{2} .
$$

The boundary conditions are

$$
\begin{aligned}
& \mathbf{R}( \pm \mathbf{L} / 2, \theta)= \pm \mathbf{E} \cos \theta \\
& +\left(\mathbf{R}_{0}^{2}-\mathbf{E}^{2} \sin ^{2} \theta\right)^{1 / 2}, \quad \mathbf{R}(\mathbf{Z}, \theta)=\mathbf{R}(\mathbf{Z}, \theta+2 \pi)
\end{aligned}
$$

which state that the liquid bridge remains anchored to the disk edges and has azimuthal periodicity, respectively.

As it will be demonstrated later, within the approximation of our study, the effects on stability limits of the considered non-axisymmetric perturbations (lateral microgravity and axes disalignment) are decoupled, that is, the stability limit variation is independent of $\alpha$. Therefore, from now on, only the case $\alpha=0$ will be considered. Observe that this implies that the microgravity direction is on the plane defined by disk axes.

Introducing the following dimensionless variables and parameters

$$
\begin{aligned}
A & =\mathbf{L} / 2 \mathbf{R}_{0}, \quad E=\mathbf{E} / \mathbf{R}_{0}, \quad B=\rho g \mathbf{R}_{0}^{2} / \sigma, \\
P & =\left(\mathbf{P}_{0}-\mathbf{P}_{\mathrm{a}}\right) \mathbf{R}_{0} / \sigma, \\
z & =\mathbf{Z} / \mathbf{R}_{0}, \quad F(z, \theta)=\mathbf{R}(\mathbf{Z}, \theta) / \mathbf{R}_{0},
\end{aligned}
$$

where $A$ is the liquid bridge slenderness, $E$ the dimensionless axes separation, $B$ the gravitational
Bond number and $P$ the dimensionless reference pressure, the formulation of the problem becomes

$$
\begin{gathered}
M[F]+P+B F \cos \theta=0, \\
\int_{-A}^{A} \mathrm{~d} z \int_{0}^{2 \pi} F^{2}(z, 0) \mathrm{d} \theta=4 \pi A, \\
F( \pm A, 0)= \pm E \cos \theta+\left(1-E^{2} \sin ^{2} \theta\right)^{1 / 2}, \\
F(z, 0)=F(z, 0+2 \pi),
\end{gathered}
$$

$M[F]$ being the dimensionless local mean curvature, $M[F]=\mathbf{R}_{0}\left(I / \mathbf{R}_{1}+1 / \mathbf{R}_{2}\right)$, which can be expressed as [8]

$$
\begin{array}{r}
M[F]=\left[F\left(I+F_{z}^{2}\right)\left(F_{v b}-F\right)+F F_{z:}\left(F^{2}+F_{\theta}^{2}\right)\right. \\
\left.-2 F_{b j}\left(F_{\theta}+F F_{z} F_{v z}\right)\right]\left[F^{2}\left(I+F_{z}^{2}\right)+F_{\theta}^{2}\right]^{-3 / 2} .
\end{array}
$$

\section{CRITICal polnts}

Critical points are given by the solution of the linear problem. Since in the base $B=0, E=0$ the system (5) - (7) has the trivial solution $F=1, P=1$, let us expand these variables in the form

$F(z, \theta)=1+\epsilon f(z, \theta)+0\left(\epsilon^{2}\right)$,

$$
P=1+\epsilon p+0\left(\epsilon^{2}\right),
$$

where $\epsilon \ll l$ is a small parameter standing for the interface deformation.

After substituting these expressions in the system (5)-(7) the following linear problem results

$$
\begin{gathered}
f+f_{z z}+f_{\theta \theta}+p=0, \\
\int_{-A}^{A} \mathrm{~d} z \int_{\theta}^{2 \pi} f(z, 0) \mathrm{d} \theta=0, \\
f( \pm A, \theta)=0, \quad f(z, 0)=f(z, 0+2 \pi) .
\end{gathered}
$$

At it is well-known[9] all solutions to this problem are axisymmetric, the expression of the interface depending on $A$. For $A=k \pi(k=1,2, \ldots)$ the interface shape results

$$
f(\bar{i}, \theta)=\sin \left(\frac{k \pi z}{A}\right), \quad p=0
$$

whereas the case $A=A_{k}$ (where $A_{k}$ satisfics $A_{k}-\tan A_{k}=0$ ) yields

$$
f(z, \theta)=-p\left(1-\frac{\cos z}{\cos A}\right)
$$

For $B \rightarrow 0, E \rightarrow 0$ the bifurcation to noncylindrical equitibrium shapes (although axisymmetric) appears only near $A=k \pi$ or $A=A_{k}$ (Implicit Function Theorem [10]). Nevertheless, only the bifurcation near $A=\pi$, which is the smallest value of $A$, is significant in practice because this point represents the transition from stable to unstable shapes. The following bifurcation points cannot be reached because the liquid bridge will break before.

Therefore, in the case $B=0, E=0$, the instability 
appears at $A=\pi$ and the unstable equilibrium shapes are defined by

$$
f(z, \theta)=\sin z, \quad p=0 .
$$

In conclusion, eqn (15) is the solution that must be perturbed to calculate the variation of the maximum stable slenderness when non-axisymmetric effects like transversal microgravity or non-coaxiality of the disks are present $(B \neq 0, E \neq 0)$.

\section{BIFURCATION EQUATION}

Since non-axisymmetric effects decrease the slenderness at which the liquid bridge becomes unstable, let us introduce, as in Rivas and Meseguer[11] a new parameter $\lambda$ measuring the slenderness decrease due to the effect of transversal microgravity or disks offset, and a new variable $x$ which normalizes boundary conditions

$$
\lambda=\frac{\pi-\lambda}{\pi}, \quad x=\frac{z}{1-\lambda} .
$$

To calculate the variation of the critical slenderness with $B$ and $E$ a standard bifurcation technique can be used[3], retaining higher order terms than those in the linear problem. Let $g(x, \theta)$ and $q$ the expressions representing these higher order terms in the expressions of the interface shape and pressure, respectively. Then, by taking into account expressions (15) the interface shape and pressure can be written as

$$
F(x, \theta)=1+\epsilon \sin x+g(x, \theta), \quad p=1+q .
$$

Therefore, after substituting expressions (16) and (17) in eqns (5)-(7) the probiem formulation becomes

$$
\begin{aligned}
& M[1+\epsilon \sin x+g(x, \theta)]+1+q \\
& \quad+B[1+\epsilon \sin x+g(x, \theta)] \cos \theta=0, \\
& \int_{-\pi}^{\pi} \mathrm{d} x \int_{\theta}^{2 \pi}[1+\epsilon \sin x+g(x, \theta)]^{2} \mathrm{~d} \theta=4 \pi^{2}, \\
& g(x, \theta)=g(x, \theta+2 \pi), \\
& g( \pm \pi, \theta)= \pm E \cos \theta+\left(1-E^{2} \sin ^{2} \theta\right)^{1 / 2} \\
& \quad-1= \pm E \cos \theta-\frac{1}{2} E^{2} \sin ^{2} \theta+\ldots
\end{aligned}
$$

One additional condition must be added to unique define the parameter $\epsilon$,

$$
\int_{-\pi}^{\pi} d x \int_{0}^{2 \pi} g(x, \theta) \sin x d \theta=0 ;
$$

observe that this condition defines $\epsilon$ as

$$
\epsilon=\frac{\int_{-\pi}^{\pi} \mathrm{d} x \int_{0}^{2 \pi} F(x, \theta) \sin x \mathrm{~d} \theta}{\int_{-\pi}^{\pi} \mathrm{d} x \int_{0}^{2 \pi} \sin ^{2} x \mathrm{~d} \theta} .
$$

The problem (18)-(21) provides $\epsilon, g(x, \theta)$ and $q$ in terms of $B, E$ and $\lambda$. As $B \rightarrow 0, E \rightarrow 0$ such functions can be calculated by means of standard perturbation techniques[12,13]. However, a direct use of this techniques requires anticipation of certain properties of the solution. This may be avoided by using the idea of the bifurcation equation( $[4,3]$. Instead of eqn (18) let us consider the following equation

$$
\begin{aligned}
M[1+\epsilon \sin x & +g(x, \theta)]+1+q+B[1+\epsilon \sin x \\
& +g(x, \theta)] \cos \theta+\phi \sin x=0 .
\end{aligned}
$$

The Implicit Function Theorem[10] shows that expressions (19)-(21) and (23) uniquely define

$$
\begin{aligned}
& \phi=\phi(\epsilon, B, E, \lambda), \\
& \quad g=g(x, \theta ; \epsilon, B, E, \lambda), \quad p=p(\epsilon, B, E, \lambda),
\end{aligned}
$$

at least when $\epsilon, B, E$, and $\lambda$ are sufficiently small. Such solutions correspond to the solution of eqns (18)-(2I) if and only if $\epsilon, B, E$ and $\lambda$ satisfy

$$
\phi(\epsilon, B, E, \lambda)=0,
$$

which is called the bifurcation equation [observe that, in this case, eqn (23) is identical to eqn (18)]

\section{BIFURCATION ORDER}

Before soiving the probiem (19)-(21) and (23) a study of the symmetries involved in the problem will allow us to anticipate some characteristics of the solution. Therefore, since the problem is invariant under the following set of symmetries

$$
\begin{aligned}
x \rightarrow & -x, \epsilon \rightarrow-\epsilon, E \rightarrow-E, \phi \rightarrow-\phi, \\
x \rightarrow & -x, \theta \rightarrow \theta+\pi, \epsilon \rightarrow-\epsilon, \\
& B \rightarrow-B, \phi \rightarrow-\phi, \\
\theta \rightarrow & \theta+\pi, B \rightarrow-B, E \rightarrow-E,
\end{aligned}
$$

and its solution is unique, the function $\phi$ must satisfy

$$
\begin{aligned}
& \phi(\epsilon, B, E, \lambda)=-\phi(-\epsilon, B,-E, \lambda), \\
& \phi(\epsilon, B, E, \lambda)=-\phi(-\epsilon,-B, E, \lambda), \\
& \phi(\epsilon, B, E, \lambda)=\phi(\epsilon,-B,-E, \lambda) .
\end{aligned}
$$

For the shake of clarity, from now on only the two simple cases $B=0, E \neq 0$ and $B \neq 0, \mathrm{E}=0$ will be considered. Let us assume $\phi(\epsilon, B, E, \lambda)$, $g(x, \theta ; \epsilon, B, E, \lambda)$ and $q(\epsilon, B, E, \lambda)$ to be expanded as

$$
\begin{aligned}
& \phi(\epsilon, B, E, \lambda)=\sum_{\substack{i j=1 \\
k, i=1}}^{\infty} \epsilon^{i} B^{\prime} E^{k} \lambda^{\prime} \phi_{i j k i} \\
& g(x, \theta ; \epsilon, B, E, \lambda)=\sum_{\substack{i, j=1 \\
k, i=1}}^{\infty} \epsilon^{i} B^{j} E^{k} \lambda^{\prime} g_{i j k k}(x, \theta), \\
& q(\epsilon, B, E, \lambda)=\sum_{\substack{i, j=i \\
k, t=1}}^{\infty} \epsilon^{i} B^{\prime} E^{k} \lambda^{l} q_{i j k t+}
\end{aligned}
$$

Then, concerning $\phi(\epsilon, B, E, \lambda)$, its Taylor expansion can be simplified taking into account eqns 
(29)-(31). Therefore, in the case $B=0, E \neq 0$ [eqnas (29) and (30)] such Taylor expansion will be

$$
\begin{aligned}
& \phi(\epsilon, 0, E, \lambda)=\epsilon \phi_{1}\left(\epsilon^{2}, E^{2}, \lambda\right) \\
& =\phi_{3000} \epsilon^{3}+\phi_{1020} \epsilon E^{2}+\phi_{100,} \epsilon \lambda+\ldots .
\end{aligned}
$$

whereas the case $B \neq 0, E=0$ [eqns (30) and (31)] yields

$$
\begin{aligned}
& \phi(\epsilon, B, 0, \lambda)=\epsilon \phi_{2}\left(\epsilon^{2}, B^{2}, \lambda\right) \\
& =\phi_{3000} \epsilon^{3}+\phi_{1200,} \epsilon B^{2}+\phi_{1000} \epsilon \lambda+\ldots
\end{aligned}
$$

Therefore, setting $\phi=0$, eqns (33) and (34) give the slenderness decrease $i$ as a function of the interface deformation $\epsilon$, the non-axisymmetric perturbation $E$ or $B$, respectively, and the corresponding coefficients $\phi_{i j k i}$. For instance, in the case $B=0$, $E \neq 0$ such expression will be

$$
\lambda=-\left(\phi_{3000} / \phi_{1001}\right) \epsilon^{2}-\left(\phi_{1020} / \phi_{1901}\right) E^{2}+\ldots
$$

This expression has been represented in Fig. 2. Since the maximum stable slenderness is reached when $\epsilon=0$, it can be concluded that critical sienderness should vary in the form

$$
\lambda_{\mathrm{c}}=-\left(\phi_{1020} / \phi_{1001}\right) E^{2}-\left(\phi_{1200} / \phi_{1001}\right) B^{2}+\ldots
$$

Observe that, since non-axisymmetric effects decrease the critical slenderness both coeflicients, $\phi_{1020} / \phi_{1001}$ and $\phi_{1200} / \phi_{1001}$, must be negative.

\section{RESULTS AND CONCLUSIONS}

When Taylor expansions (32) are introduced into equs (19)-(2i) and (23) and the coefficient of each monomial $\epsilon^{i} B^{j} E^{k} \lambda^{\prime}$ is set to zero, a recursive sequence of hinear problems results, which allow calculation of $\phi_{i j k i}, q_{i j k j}$ and $g_{i j k i}(x, \theta)$. Then, the following results are obtained

$$
\begin{aligned}
& g_{1000}(x, \theta)=\sin x, \\
& g_{2000}(x, \theta)=-\frac{1}{4}+\frac{1}{4} \cos 2 x, \\
& g_{0001}(x, \theta)=0,
\end{aligned}
$$

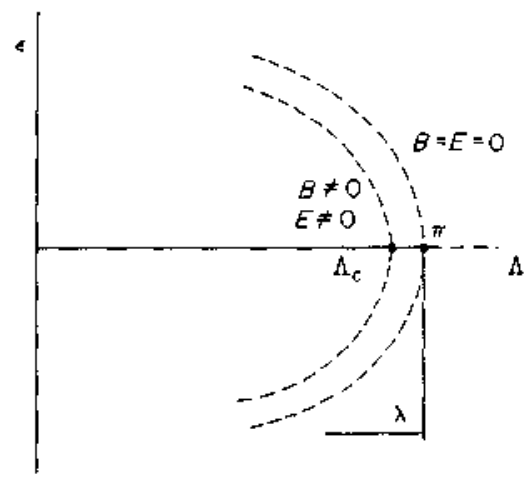

Fig. 2. Bifurcation diagram. Liquid bridge deformation $c$ vs sienderness $A$. This plot shows the influence of the dimensionless iransversal microgravity $B$ or disk offset $E$ in the bifurcation.

$$
\begin{aligned}
g_{0010}(x, \theta)= & \frac{x}{\pi} \cos \theta \\
g_{1010}(x, \theta)= & \frac{1}{\pi} \cos \theta(1+\cos x), \\
g_{0020}(x, \theta)= & \frac{1}{4 \pi^{2}} \cos 2 \theta\left(x^{2}+1-\frac{\cosh \sqrt{3} x}{\cosh \sqrt{3} \pi}\right) \\
& -\frac{1}{4 \pi^{2}} x^{2}, \\
g_{0100}(x, 0)= & \frac{1}{2}\left(\pi^{2}-x^{2}\right) \cos 0, \\
g_{1100}(x, \theta)= & -\frac{1}{2}(x \cos x+x-3 \sin x) \cos \theta, \\
g_{0200}(x, \theta)= & -\frac{1}{16}\left(\pi^{2}-x^{2}\right)^{2}+\frac{3}{4}\left(x^{2}+\pi^{2} \cos x\right) \\
& -\frac{\pi^{2}}{4}(1+\cos x)+\left[\frac{1}{16}\left(\pi^{2}-x^{2}\right)^{2}\right. \\
& +\frac{1}{6}\left(1-\frac{\cosh \sqrt{3} x}{\cosh \sqrt{3} \pi}\right) \\
& \left.+\frac{3}{4}\left(x^{2}-\pi^{2} \frac{\cosh \sqrt{3} x}{\cosh \sqrt{3} \pi}\right)\right] \cos 2 \theta ;
\end{aligned}
$$

$$
\begin{aligned}
& \phi_{1000}=\phi_{2000}=\phi_{0001}=\phi_{00032}=\phi_{0010}=\phi_{1010}=0, \\
& \phi_{0011}=\phi_{00220}=\phi_{01000}=\phi_{0101}=\phi_{1100)}=\phi_{0200}=0, \\
& \phi_{3000}=-\frac{3}{2}, \quad \phi_{1001}=2, \\
& \phi_{1020}=-\frac{3}{2 \pi^{2}}, \quad \phi_{12000}=-\frac{\pi^{2}}{2},
\end{aligned}
$$

where most of these cocfficients have been directly canceled, as resulting from the symmetries analysis presented in Section 5. Therefore, accoroling to expressions (38) when non-axisymmetric effects are considered the critical slenderness decreases in the form

$$
\lambda_{c}=\frac{3}{4 \pi^{2}} E^{2}+\frac{\pi^{2}}{4} B^{2}+\ldots
$$

which coincides with the result obtained by Coriell et $a l .[4]$ in the case $B \neq 0, E=0$.

An important characteristic to be pointed out is that the results here obtained do not depend on the angle between the plane defined by the axes of the disks and the direction of transversal microgravity (see Fig. 1), so that eqn (39) remains the same although this angle was not equal to zero. In consequence, it must be noted that there is no coupling between the two considered non-axisymmetric perturbations, that is, the term of order $\mathrm{BE}$ is equal to zero.

In conclusion, it has been demonstrated that, in the two cases analyzed, branching is suberitical, and that a cylindrical long liquid bridge will be stable when its slenderness is smaller than the critical slenderness

$$
A_{\mathrm{c}}=\pi\left(1-\frac{3}{4 \pi^{2}} E^{2}-\frac{\pi^{2}}{4} B^{2}+\ldots\right)
$$


On the other hand, the expression for the stable equilibrium shapes is

$$
\begin{aligned}
F(x, \theta)=1 & +E_{g_{0010}}(x, \theta)+E^{2} g_{0020}(x, \theta) \\
& +B g_{0100}(x, \theta)+B^{2} g_{0200}(x, \theta)+\ldots
\end{aligned}
$$

where the functions $g_{i j k}$ are given by the expressions (37).

Acknowledgements - This work has been supported by the Spanish National Commission for Space Research (CONIE). I wish to thank Dr J. Meseguer and Dr J. M. Vega for their hejpful discussions.

\section{REFERENCES}

1. J. Meseguer and A. Sanz, Numerical and experimental study of the dynamics of axisymmelric liquid bridges. $J$. Fiuid Mech. 153, 83-101 (1985).

2. R. A. Brown and $L$. E. Scriven. The shapes and stability of captive rotaling drops. Phil. Trans. R. Soc. Lond. 297, $51-79(1980)$.

3. J. M. Vega and J. M. Perales, Almost cylindrical isorolating liquid bridges for small Bond numbers. In Materials Sciences Under Microgravity, ESA SP-191. ESA, Paris, pp. 247-252 (1983).
4. S. R. Coriell, S. C. Hardy and M. R. Cordes, Melt shape in weightless crystal growth. NBS Space Processing Research, NBSIR 76-980 (1976)

5. S. R. Coriell, S. C. Hardy and M. R. Cordes, Stability of liquid zones. J. Colloid Interface Sci. 60, 126-136 (1977).

6. I. Martinez, Liquid column stability. In Marerial Sciences Under Microgravity, ESA SP.222, ESA, Paris, pp. 31-36 (1984)

7. J. Meseguer, Stability of long liguid columns. In $\mathrm{Ma}$ serial Sciences Under Microgravify, ESA SP-222, ESA, Paris, pp. 297-300 (1984).

8. D. J. Struik, Classical Differential Geomesry. AddisonWesley, New York (1957).

9. I. Martinez, Stability of axisymmetric liquid bridges. In Materials Sciences Under Microgravity, ESA SP-[91, ESA, Paris, pp. 267-273 (1983).

10. S. Lang, Real Analysis. Addison-Wesley, New York (1969).

1I. D. Rivas and J. Meseguer, One-domensional, selfsimilar solution of the dynamics of axisynmetric slender liquid bridges. J. Fluid Mech. 138, 4l7-4l9 (1984).

12. M. H. Millmann and J. B. Keller, Perturbation theory of nonlinear boundary-value problems. J. Math. Phys. 10, 342-36! (J969).

13. B. J. Matkowsky and E. L. Reiss, Singular perlurbations of bifurcations. SIAM J. Appt. Math. 33, $230-255(1977)$

14. S. N. Chow and J. K. Hale, Methods of Bifurcation Theory. Springer, Beritin (1982). 